Acta Crystallographica Section B

Structural

Science

ISSN 0108-7681

\section{Frank H. Allen}

Cambridge Crystallographic Data Centre, 12 Union Road, Cambridge CB2 1EZ, England

\section{Structural Science Online}

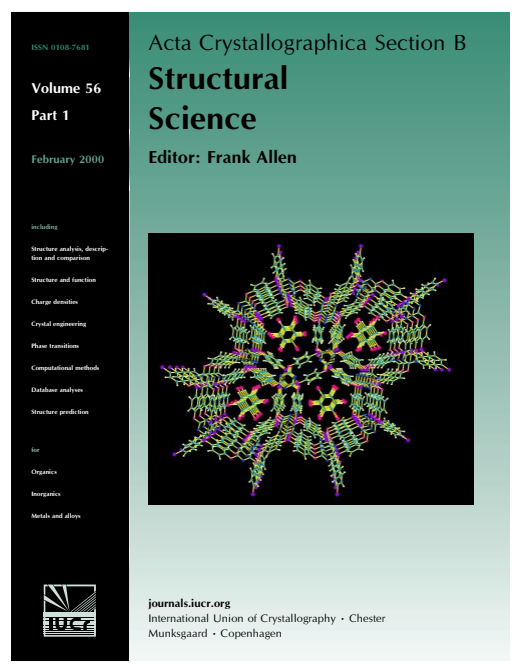

\section{Structural Science enters the electronic era}

Readers will notice that the format and presentation of Section B of Acta Crystallographica have been upgraded from this first issue of 2000. This continues a policy of stylistic enhancements to all sections of Acta that reflect improved technological capabilities. These changes are closely related to the ongoing electronic revolution in the preparation and publication of IUCr journals, and the launch of IUCr's Crystallography Online initiative. For Section $B$ this means that:

(a) Notes for Authors and the Transfer of Copyright Agreement can be downloaded from the Web at http://journals.iucr.org/b/services/authorservices.html.

(b) Electronic submission (diskette or e-mail) of final manuscripts is preferred.

(c) Most papers are typeset in-house at Chester and proofs are available electronically.

(d) Full-text versions of recent volumes are web-accessible to subscribers.

(e) Web access to full-text versions of all Acta papers is planned.

Crystallography Online offers a range of other user services and these are described at http://journals.iucr.org.

These upgrades are, of course, changes of style rather than of scientific substance. Section $B$ remains committed to the publication of high-quality articles across the diverse spectrum of crystallographic research that is designated as Structural Science. The thumbnail phrases now included on the cover express principal themes in this diversity. Readers should note that full discussions of sets of related crystal strucures are encouraged in Section B, and extended descriptions of individual structures are acceptable where the length of the discussion is scientifically warranted, and exceeds that which may be published elsewhere in IUCr journals.

The types of papers published by Section $B$ also remain unchanged. Full Research Papers remain the central feature of the journal, complemented by Short Communications, Topical Reviews, Lead Articles and Letters to the Editor. Submission details are fully described in the updated Notes for Authors. Section B is committed to the publication of high-quality review material across its sphere of interest. Topical Reviews of up to 10 journal pages provide for focused surveys of 'hot topics' in current research, while Lead Articles provide for longer in-depth accounts of major developments. Review material is commissioned by the Section Editor after discussion of scope and content with potential authors. Suggestions for both Topical Reviews and Lead Articles are welcomed.

We hope that the stylistic enhancements, coupled with high-quality scientific content, make Section $B$ an attractive publication medium. Rapid advances in electronic production methods should significantly increase the throughput of articles and make them more easily accessible worldwide. Through these changes, Section $B$ hopes to expand its appeal to authors and readers alike. 